EXTENDED REPORT

\title{
Orbital exenteration: a 13 year Manchester experience
}

\author{
I Rahman, A E Cook, B Leatherbarrow
}

Br J Ophthalmol 2005;89:1335-1340. doi: 10.1136/bjo.2004.062471

See end of article for authors' affiliations

.....................

Correspondence to: Imran Rahman, Manchester Royal Eye Hospital, Lister Centre, Nelson Street, Manchester M13 9WL, UK;

imran1973@tiscali.co.uk

Accepted for publication 3 February 2005

\begin{abstract}
Background/aims: Orbital exenteration is a psychologically and anatomically disfiguring procedure reserved for the treatment of potentially life threatening malignancies or relentlessly progressive conditions unresponsive to other treatments. In this study the authors aimed to review their experience with exenteration, including indications, outcomes, and reasons for the increased rate of exenterations over the past 15 months.

Method: This retrospective study reviewed operating department records via a computerised database to identify all patients who had undergone exenteration of the orbit from 1 January 1991 to 1 April 2004 inclusive, at the Manchester Royal Eye Hospital. Where case records were unavailable, attempts were made to obtain patient data from general practitioners, local health authorities, and referring hospitals. Results: 69 orbits of 68 patients were identified. The mean age of the cohort was 68.2 years, with 33 males and 35 females having undergone exenterations. In total, 31 patients had previously undergone treatments undertaken by the referring specialty with a mean time from the primary procedure to exenteration of 115 months. 14 different tumours were encountered, of which basal cell carcinoma (28), melanoma (10), sebaceous cell carcinoma (nine), and squamous cell carcinoma (six) were the most common. An increasing incidence was observed in cases of BCCs requiring exenteration. 30 patients received orbital prosthesis within an 11 month period post-exenteration.

Conclusion: Exenteration is a procedure performed with increasing frequency in this unit over the past 15 months, the majority the result of BCCs. A large proportion of these exenterations had undergone previous treatments under a variety of non-ophthalmic specialties in other units. Exenterations are disfiguring procedures that may, therefore, be reduced in incidence by aggressive removal at the time of primary removal. Once performed, the cosmetic rehabilitation is long, with multiple postoperative visits, independent of the method used to close the orbital defect.
\end{abstract}

\section{METHODS AND SUBJECTS}

This retrospective study reviewed operating department records via a computerised database to identify all patients who had undergone exenteration of the orbit from 1 January 1991 to 1 April 2004, inclusive, at the Manchester Royal Eye Hospital. All surgical records were examined to determine indications for exenteration, referral methods, diagnosis and surgical treatment elsewhere, location and extension of pathological lesions, and histological diagnosis. In the case of orbital and periorbital malignancies the histological reports were examined to determine whether or not clear histological margins had been obtained. Surgical technique, adjunctive postoperative treatments (further surgery, radiotherapy, chemotherapy), intraoperative and postoperative complications, recurrences, choice of aesthetic camouflage (occlusive patch, opaque spectacle lens, orbitofacial prosthesis), and time from exenteration to rehabilitation of the facial disfigurement with a formal prosthesis were also noted. Recurrence of any malignant disease was also noted.

Where surgical notes were unavailable, data were compiled from correspondence letters from the department of ocular prosthetics, general practitioners, and the referring hospital records.

\section{RESULTS}

Between I January 1991 to 1 April 2004, 69 orbits of 68 patients were identified (one patient having bilateral exenterations for severe contracted sockets secondary to

Abbreviations: BCC, basal cell carcinoma; SCC, squamous cell carcinoma 


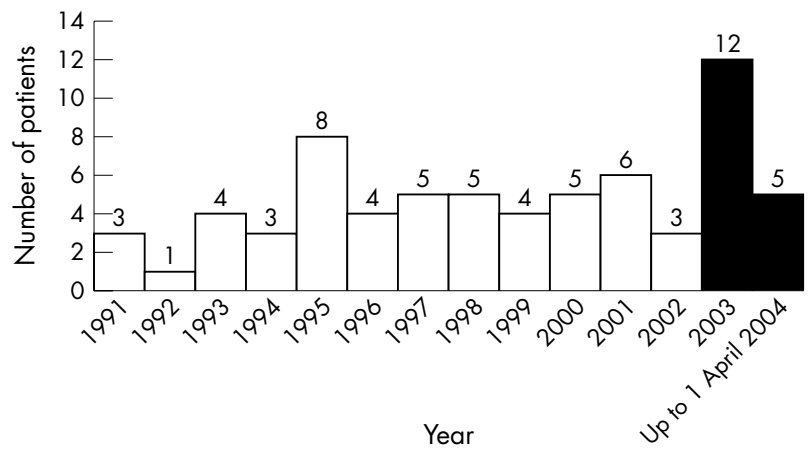

Figure 1 Distribution of exenteration patients over 13 year period.

Stevens-Johnsons syndrome) from the theatre database as having undergone orbital exenteration (fig l). Case notes for 57 patients were available for review. Histological data, letters from general practitioners, and clinic letters were used to collate the data for the remaining 12 patients. A total of 35 females and 33 males made up the study group, with 35 right orbits and 34 left orbits being affected. The mean age of the cohort was 68.2 years (median of 72, range 4-91 years), at the time of exenteration.

Preoperative visual acuity was unavailable in 18 patients; 21 of the remaining 51 patients $(41 \%)$ had a Snellen visual acuity of $6 / 12$ or better. In the eye not exenterated, Snellen visual acuity of 6/12 or better was observed in 41 out of 53 eyes $(77 \%)$, in whom visual acuity measurements were available preoperatively.

Five orbits of four patients underwent exenteration for non-malignant disease. One patient had a chronic discharging socket, another a contracted socket, and the third (two eyes) had severe Stevens-Johnson syndrome. The last patient had severe chemical injury necessitating facial cosmetic rehabilitation. This report deals chiefly with those patients with neoplastic indications for exenteration, and these five patients with non-neoplastic conditions are not considered further, except where surgical technique and complications are concerned. An overview of indications for exenteration is presented in table 1 .

Although the surgery was carried out by a senior ophthalmic surgeon in all cases, referrals were made from varying specialties including dermatology, plastic surgery, neurosurgery, ENT, oncology, and from general practitioners. In total, nine patients had previous treatment in Manchester and a further 31 patients had previously undergone treatments undertaken by the referring specialty. Sixteen had had a local tumour excision, one had undergone orbital radiotherapy, and a further 10 had had a combination of surgery and orbital radiotherapy. Two patients proceeded to orbital exenteration following failed attempts at Mohs' micrographic surgical excision of extensive periocular malignancies. Of the final two patients, one underwent enucleation and the other multiple incisions with curettage. The mean time from the

Table 1 Indications for orbital exenteration

\begin{tabular}{ll}
\hline Indication & $\begin{array}{l}\text { Number of patients } \\
(\mathbf{n = 6 9 )}\end{array}$ \\
\hline Lid neoplasm & 38 \\
Orbital neoplasm & 18 \\
Non-neoplastic & 5 \\
Conjunctival neoplasm & 3 \\
Globe neoplasm & 2 \\
Unknown & 3 \\
\hline
\end{tabular}

Table 2 Previous therapy

\begin{tabular}{ll}
\hline Therapy & $\begin{array}{l}\text { Number of patients } \\
(\mathbf{n = 6 4 )}\end{array}$ \\
\hline None & 24 \\
Local excision & 16 \\
Local excision and radiotherapy & 10 \\
Mohs' abandoned & 2 \\
Radiotherapy alone & 1 \\
Unknown/not documented & 8 \\
Other & 3 \\
\hline
\end{tabular}

primary procedure to exenteration was 115 months (range 1600 months). Treatment modalities undertaken before exenteration are outlined in table 2.

Table 3 shows the histological diagnosis of the tumour. Fourteen different tumours were encountered, of which basal cell carcinoma (BCC) (28), melanoma (10), sebaceous cell carcinoma (nine) and squamous cell carcinoma (SCC) (six) were the most common. The remaining 10 tumour types accounted for the other 11 cases.

Seventeen exenterations have been performed over a 15 month period since the beginning of 2003. Of these, 11 $(65 \%)$ had orbitally invasive BCC. In the previous 12 years a total of 47 exenterations were performed, 17 (36\%) of which had orbital invasion by BCCs. It, therefore, appears that invasive BCC as an indication for orbital exenteration has increased. It is also interesting that $39 \%$ in the group with exenterations over 12 years compared to an increased $69 \%$ in the group in the most recent 15 months had at least one previous attempt at cure of the lesion. This may imply a trend towards less destructive surgical techniques, resulting in inadequate surgical clearance of the tumour or inappropriate follow up before exenteration is undertaken.

The anatomical location of these lesions is outlined in table 4 . The eyelids were the most common sites of tumour, occurring in 38 of the 64 exenterated patients. Malignant disease originating in the orbit and lacrimal gland accounted for a further 18 cases, with the globe and conjunctiva accounting for a further five cases.

Table 3 Histological diagnosis of patients undergoing exenteration

\begin{tabular}{ll}
\hline Histological diagnosis & $\begin{array}{l}\text { Number of } \\
\text { patients }(\mathbf{n}=69)\end{array}$ \\
\hline Basal cell carcinoma & 9 \\
with no previous treatment & 10 \\
with previous surgery only & 8 \\
with previous radiotherapy/surgery & 1 \\
with previous radiotherapy & 9 \\
Sebacous cell carcinoma & 6 \\
Squamous cell carcinoma & \\
Melanoma & 8 \\
$\quad$ Globe with extrascleral spread & 1 \\
Lids & 1 \\
Ethmoid sinuses & 2 \\
Adenocystic carcinoma (lacrimal gland) & 1 \\
Adenocarcinoma & 1 \\
Liposarcoma & 1 \\
Merkel cell & 1 \\
Lymphoma & 1 \\
Apocrine carcinoma & 1 \\
Rhabdomyosarcoma & 1 \\
Secondary metastasis-gastrointestinal primary & 1 \\
Malignant schwannoma & 1 \\
Malignant medulloepithelioma & 5 \\
Non-malignant disease & \\
\hline
\end{tabular}




\begin{tabular}{|ll|}
\hline Table 4 Location of lesion \\
\hline Origin of lesion & $\begin{array}{l}\text { Number of patients } \\
(\mathbf{n}=64)\end{array}$ \\
\hline Lids & \\
Medial canthus & 17 \\
Upper lid & 8 \\
Lower lid & 8 \\
Lateral canthus & 5 \\
Orbital & 16 \\
Lacrimal gland & 2 \\
Globe & 2 \\
Conjunctiva & 3 \\
Other/unknown & 3 \\
\hline
\end{tabular}

In the overall total of 68 patients (69 orbits) undergoing orbital exenteration, including those for non-malignant disease, 25 required a subtotal exenteration and a further 35 a total orbital exenteration. In nine cases the procedure was not clearly documented or the information was not retrievable. Surgery was performed under local anaesthesia with intravenous sedation in seven cases and under general anaesthesia in the remaining 62 cases. Patients were selected for local anaesthetic with sedation on the basis of co-existing medical problems, in whom general anaesthesia was deemed unsafe following advice from a consultant anaesthetist.

In three of the 64 cases requiring exenteration for malignant disease, it was deemed necessary to remove part of the bony orbit to ensure tumour clearance at the time of surgery. Overall, 29 patients demonstrated tumour to be present in excision margins on histological examination following exenteration. Nineteen of these 29 underwent further postoperative adjunctive treatment; 16 underwent orbital radiotherapy, one patient required a combination of radiotherapy and chemotherapy, one patient needed radiotherapy and further surgery, and one patient required further removal of tissue to achieve histologically clear margins.

The orbit was allowed to granulate in a total of 43 of the 64 patients. In a further 21 a split skin graft was applied. Postoperatively the most common complication was sinoorbital fistulas occurring in 15 patients. These occurred in 12 patients in whom the orbit was allowed to granulate and in only three patients in whom a split skin graft was placed. Fistula, therefore, developed more commonly with sockets left to granulate $(80 \%)$ than with the concomitant use of skin grafts $(20 \%)$. Seven further patients had graft failure, four of whom required regrafting and three others were allowed to granulate. A cerebrospinal fluid leak was observed intraoperatively in one patient who had previously undergone a craniotomy, but this was managed at the time of the exenteration with a local pericranial flap with no further adverse sequelae. Another patient had chronic socket discharge in the absence of a fistula. These complications and co-morbidity are detailed in table 5.

Table 5 Complications and co-morbidity experienced in the 64 patients undergoing exenteration for neoplasm

\begin{tabular}{ll}
\hline Complication/comorbidity & $\begin{array}{l}\text { No of patients } \\
(\mathbf{n}=64)\end{array}$ \\
\hline None & 35 \\
Sino-orbital fistula & 15 \\
Failed skin graft requiring regrafting & 4 \\
Failed skin graft allowed to granulate & 3 \\
Chronic discharge & 1 \\
Cerebrospinal fluid leak & 1 \\
Complications not documented & 5 \\
\hline
\end{tabular}

There were eight cases of local or regional recurrence of tumours, outlined in table 6. This group included two SCCs, one liposarcoma, one SCC, one BCC, one melanoma, one adenocarcinoma from a gastrointestinal tumour, and one adenocarcinoma occurring in a lacrimal gland pleomorphic adenoma. Of these eight cases, six patients had tumour present on histological examination. Two had clear resection margins. Seven of the eight patients underwent adjunctive radiotherapy postoperatively.

In total, 31 patients had facial prostheses fitted at an average of 10 months (range 2-38 months) postoperatively (fig 2). Of these, four patients underwent successful 2 stage osseointegration before prosthesis fitting. There was no difference in the mean time to prosthesis fitting whether or not a split skin graft was applied (11 months for orbits allowed to granulate compared to 10 months using a skin graft). However, 25 patients were satisfied with the cosmetic outcome without a facial prosthesis and opted for a "pirate" patch to cover the exenterated defect. A further eight patients had not completed prosthesis fitting at the time of writing.

Of the five orbits exenterated for non-malignant disease, four were satisfied with the cosmetic outcome without facial prosthesis. One further orbit had prosthesis fitting 3 months after exenteration with the application of a skin graft.

A complete review of morbidity and mortality is beyond the scope of this paper and will be presented in a follow up study.

\section{DISCUSSION}

Orbital exenteration is a disfiguring procedure requiring preoperative and postoperative counselling by a multidisciplinary team consisting of an ocularist, orbital surgeon, specialist wound care nurse, and a clinical psychologist. Preoperative counselling with an ocularist is pivotal in deciding on the timing and best surgical approach-for example, whether osseointegration at the time of exenteration and before radiotherapy will save the patient later need for hyperbaric oxygen. Tumour eradication, however, should take precedence over cosmetic concerns. Often, removal of part of the bony orbit is necessary adding to the postoperative facial rehabilitation management plan. Recently, we have adapted the successful use of intravenous sedation with local anaesthetic (seven cases) as a means of exenterating the older patient with systemic co-morbidity.

Although non-malignant disease may occasionally be the indication for orbital exenteration, the vast majority of exenterations are undertaken to treat malignant disease. Bartley et al $^{10}$ reported that 100 of their 102 exenterated cases resulted from malignant disease. This is similar to Levin et al (93 of 99 cases) $)^{9}$ and Mohr and Esser (74 of 77 cases). ${ }^{6}$ We found a similar ratio in our series of 64 of 69 cases requiring exenteration for malignant disease.

Healing of the orbital defect is dependent on the method used for reconstruction. Options include temporalis muscle transposition, ${ }^{11}$ midline forehead flaps, ${ }^{12}$ dermal graft, ${ }^{13}$ dermis fat graft, ${ }^{14}$ split skin graft, ${ }^{15}$ globe sparing exenteration, ${ }^{16}$ eyelid sparing techniques ${ }^{17}$ and spontaneous granulation. ${ }^{18}$ Allowing granulation of the defect may delay healing by up to 2-3 months. The need for regular dressing changes must be weighed against the potential benefits of healing by secondary intention-namely, an easier, quicker procedure that allows recurrences to be detected early. This provides a comparable colour match to the surrounding skin when granulation is complete. ${ }^{10}$ In our practice, postoperative dressing changes are undertaken by experienced nurse practitioners using a moist wound bed healing technique. The orbit is packed with Aquacel (Convatec Ltd, Deeside, UK), and dressings changed on a weekly basis until complete healing of the orbit. 
Table 6 Characteristics of patients with local or regional recurrence

\begin{tabular}{|c|c|c|c|c|c|c|}
\hline Age/sex & Diagnosis & Clear margin & Adjuvant treatment & Previous treatment & Cause of death & Comment \\
\hline $70 / M$ & Sebaceous cell & No & Radiotherapy & No & Secondary metastases & $\begin{array}{l}\text { Had previous } \\
\text { radiotherapy }\end{array}$ \\
\hline $27 / F$ & Liposarcoma & No & Radiotherapy & No & Secondary metastases & Local bone involvement* \\
\hline $80 / M$ & Metastases & No & None & No & Secondary metastases & Gastrointestinal primary \\
\hline $74 / M$ & Sebaceous cell & No & Radiotherapy & Surgery & Secondary metastases & Delayed diagnosis. \\
\hline $85 / F$ & BCC & No & Radio/surgery & Radio/surgery & Alive & $\begin{array}{l}\text { Cryotherapy for } \\
\text { recurrence }\end{array}$ \\
\hline $72 / M$ & $\begin{array}{l}\text { Melanoma with } \\
\text { extrascleral spread }\end{array}$ & Yes & Radiotherapy & Radio/surgery & Unknown status & $\begin{array}{l}\text { Neck metastasis at last } \\
\text { visit }\end{array}$ \\
\hline $72 / F$ & SCC & No & Radio/surgery & No & Secondary metastases & Local bone involvement* \\
\hline $63 / M$ & $\begin{array}{l}\text { Adenocarcinoma in } \\
\text { lacrimal gland } \\
\text { pleomorphic adenoma }\end{array}$ & Yes & Radiotherapy & Radio/surgery & Secondary metastases & Local bone involvement ${ }^{\star}$ \\
\hline
\end{tabular}
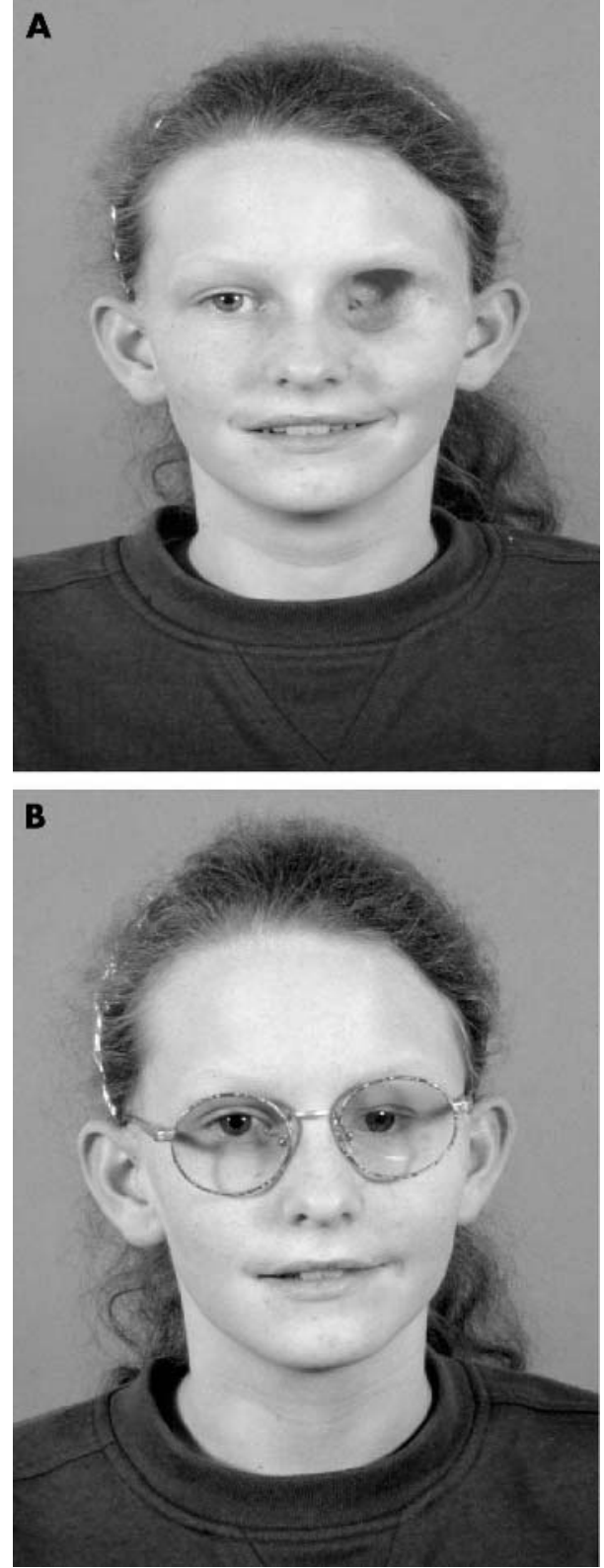

Figure 2 (A) Patient before facial prosthesis. (B) Patient with facial prosthesis. (Pictures reproduced with permission of patient and her parents.)
Significant numbers of patients may develop sino-orbital fistulas from perforated sinuses occurring intraoperatively. In our series, 12 of the $43(28 \%)$ orbits allowed to granulate developed permanent fistula formation following granulation of the orbit. This is a lower rate when compared to other large studies, $17 / 25$ (68\%) cases $^{6}$ and $17 / 49$ (35\%) cases. ${ }^{10}$ Other disadvantages of secondary healing of an exenterated orbit include delayed healing, prolonged postoperative socket care, and delayed facial rehabilitation. The method employed to close the orbital defect must take into account several important issues: local and systemic tumour control and the likely survival of the patient. For instance, in cases where recurrence is unlikely and survival good, granulation may be beneficial to allow local detection of tumour. Conversely, in cases where exenteration is preformed for palliation of the neoplasm, a split skin graft will give rapid rehabilitation of the socket, and minimise hospital attendance. The use of such a graft, however, lengthens the duration of surgery and can have morbidity in older patients. Furthermore, in our study, there was no significant difference in the time to facial rehabilitation with prosthesis for those orbits allowed to granulate (11 months) compared to those orbits in which a split skin graft was applied (10 months).

In our series, BCC was the most common neoplasm (44\%), followed by melanoma of the eyelids, sinuses, and globe with extrascleral spread, and then SCC. Combined, these three tumours accounted for $73 \%$ of cases requiring exenteration. Other large studies have reported varying rates of BCC. However, our rate of $44 \%$ is significantly higher. Rathbun et $a l^{8}$ reported 14 cases of BCC in their series of $48(29 \%)$ exenterations, Bartley et al ${ }^{10} 21$ of $100(21 \%)$ cases, Mohr and Esser $^{6}$ six of $74(8 \%)$ exenterations, and eight of $99(8 \%)$ cases reported in the series by Levin and Dutton. ${ }^{9}$ Two cases of orbital exenteration in our BCC group had Gorlin-Goltz syndrome, $^{19}$ one of which underwent exenteration for palliative reasons.

It is interesting to note that $23 / 28(82 \%)$ cases of those orbits exenterated for orbital invasion of a BCC had previously experienced failed treatments (surgery/radiotherapy) elsewhere. This was similar to the overall figure documented by Bartley et al of $80 \% .^{10}$ More recently, there has been a significant increase in the number of exenterations being undertaken at our institution for orbital invasion by a BCC. Since 1 January 2003, 11/17 cases of orbital invasive BCC have undergone exenteration compared to 17/ 47 cases in the previous 12 years. Further, in the most recent 15 months $69 \%$ of patients have had treatment of neoplastic lesions before exenteration. In comparison, in those with exenteration over a 12 year period, 39\% received previous attempts at tumour removal before referral for exenteration. There has been a trend towards a greater number of patients 
being treated before exenteration over the past 15 months $(69 \%)$ compared to the previous 12 years $(39 \%)$.

Since 2001 , almost $50 \%$ of cases with orbitally invasive BCCs have been treated and referred by allied specialtiesthat is, plastic surgery, dermatology, and oncology, and the remaining $50 \%$ or so by regional ophthalmologists. Preceding this period, however, the majority $(70 \%)$ had been treated and referred by ophthalmologists. This may reflect the more diverse interest of eyelid tumour surgery among allied specialties, often using non-Mohs' micrographic techniques, resulting in recurrence of tumours.

A high rate of recurrence is observed in medial canthal tumours and was confirmed in our series where 12 of 23 cases of recurrence were from medial canthal lesions, necessitating exenteration. ${ }^{20}$ Only one case of BCC had recurrence after Mohs' micrographic surgery. This may reflect the medial canthal area as an area where BCCs are common or a site where recurrence and invasion may be prominent. Many of these cases may have avoided exenteration if treated early; factors that are likely to have influenced their outcome include inappropriate surgery, inappropriate observation and/ or follow up, and neglect of the lesion by the patient until a late stage in the disease.

Management of uveal tumours remains controversial, but it is now generally agreed that primary exenteration does not increase survival rates and may even hasten progress of the disease process. ${ }^{21-26}$ In our series, there was a $100 \%$ survival after 1 year. The 3 year survival fell to $33 \%$, although only six patients have been followed up for this length of time. Furthermore, three patients had an exenteration for palliation of symptoms.

Two schools of thought exist when deciding on exenteration for these highly malignant uveal tumours with extrascleral spread. Those opposing exenteration think that the highly metastatic nature of these tumours necessarily involve micro-metastases when extrascleral extension is identified and, thus, carry a poor prognosis with or without treatment. Those advocating exenteration believe that removing the tumour as early as possible may increase survival by limiting the time for systemic spread to occur. Shammas and Blodi ${ }^{23}$ reported an overall $73 \%$ mortality of patients when extrascleral extension of the tumour was observed, compared to $22 \%$ when extrascleral extension was not observed. Kersten et $a^{27}$ found a similar tumour related mortality of $81 \%$ in patients undergoing extrascleral extension. Furthermore, there was no clinically significant difference in overall survival probabilities of patients undergoing early exenteration $(21 \%)$ compared to enucleation ( $17 \%)$ alone on extended follow up, and a $100 \%$ incidence of metastasis related to orbital recurrences needing only palliation. Thus, the preferred treatment for extrascleral extension of uveal melanomas appears to be local resection of the tumour, including enucleation and tenonectomy.

Sebaceous gland carcinoma of the eyelid is a slow growing lesion, often mimicking benign conditions, such as a chalazion. ${ }^{28}$ Of the eight cases in our series, only one had had previous surgery on the assumption that the lesion was a chalazion. After several recurrences over a 24 month period before referral, a biopsy confirmed sebaceous cell carcinoma. However, the remaining seven patients had biopsy confirmation without previous surgery elsewhere. All had confirmed pagetoid conjunctival spread. A mortality rate of 3-40\% has been reported..$^{29-34}$ Early diagnosis can, however, prevent mutilating exenteration and may be amenable to less destructive surgery. We found a mortality rate of $25 \%$ (two patients) in this subgroup from metastatic disease and a further one patient with metastatic disease, but having palliative treatment. All three of these patients did not achieve clear surgical margins at the time of exenteration.

\section{CONCLUSION}

Exenteration is a procedure performed with increasing frequency in our unit over the past 15 months, the majority because of BCCs. The incidence of $44 \%$ of exenterations at our unit being performed for orbitally invasive BCC is significantly higher than other large studies; 39\% of these were performed in the past 15 months. A large number of patients with these exenterations had undergone previous treatments under a variety of non-ophthalmic specialties in other units, using non-Mohs' micrographic techniques. Coupled with possible inappropriate observation and/or follow up, or neglect of the lesion by the patient, eyelid tumours are presenting at an advanced stage.

Exenterations are disfiguring procedures that may, therefore, be reduced in incidence by primary removal of tumours by those most skilled at tumour removal. Once performed, the cosmetic rehabilitation is long, with multiple postoperative visits, independent of the method used to close the orbital defect. Our rate of fistula formation is significantly lower than other reports, which may reflect surgical technique. Further, we have found the use of local anaesthetic with sedation to be very effect in specific patients with medical co-morbidity without difficulties during exenteration.

\section{Authors' affiliations}

I Rahman, A E Cook, B Leatherbarrow, Manchester Royal Eye Hospital, Lister Centre, Nelson Street, Manchester M13 9WL, UK

Competing interests: none declared

\section{REFERENCES}

1 Bartisch G. Ophthalmodoulcia, Dresden, 1583:3:208.

2 Rose GE, Wright JE. Exenteration for benign orbital disease. $\mathrm{Br} J$ Ophthalmol 1994;78:14-18.

3 Sheilds JA, Sheilds CL, Suvarnamani C, et al. Orbital exenteration with eyelid sparing: indications, technique and results. Ophthalmic Surg 1991;22:292-7.

4 Frezotti R, Nuti A. Repair after orbital exenteration. Acta Neurochir 1982;60:119-24

5 Shore JW, Burks WR, Leone C, et al. Dermis fat graft for orbital exenteration after subtotal exenteration. Am J Ophthalmol 1986;102:228-36.

6 Mohr C, Esser J. Orbital exenteration: surgical and reconstructive strategies. Graefes Arch Clin Exp Ophthalmol 1997;235:288-95.

7 Naquin HA. Exenteration of the orbit. Arch Ophthalmol 1954;51:850-62.

8 Rathbun JE, Beard C, Quickert MH. Evaluation of 48 cases of orbital exenteration. Am J Ophthalmol 1971;72:191-9.

9 Levin PS, Dutton JJ. A 20 year series of orbital exenteration. Am J Ophthalmol $1991 ; 112: 496-501$

10 Bartley GB, Garrity JA, Waller RR, et al. Orbital exenteration at the Mayo Clinic 1967-1986. Ophthalmology, 1989;96;468-74.

11 Reese $A B$, Jones IS. Exenteration of the orbit and repair by transplantation of the temporalis fascia. Am J Ophthalmol 1961;51:217-27.

12 Dortzbach R, Hawes M. Midline forehead flap in reconstructive procedure of the eyelids and exenterated socket. Ophthalmic Surg 1981;12:257-68

13 Mauriello JA Jr, Han KH, Wolfe R. Use of autogenous split-thickness dermal graft for reconstruction of the lining of the exenterated orbit. Am J Ophthalmol 1985; 100:465-7

14 Shore JW, Burks R, Leone CR Jr, et al. Dermis fat graft for orbital reconstruction after subtotal exenteration. Am J Ophthalmol $1986 ; 102: 228-36$

15 Harting F, Koorneef L, Peeters HJF, et al. Glued fixation of split-skin graft to the bony orbit following exenteration. Plast Reconstruc Surg 1985;76:633-5.

16 Catalano PJ, Laidlaw D, Sen C. Globe sparing orbital exenteration. Otolaryngol Head Neck Surg 2001;125:379-84

17 Sheilds JA, Sheilds CL, Suvarnamani C, et al. Orbital exenteration with eyelid sparing: indications, technique and results. Ophthalmic Surg
$1991 ; 122: 292-7$.

18 Putterman AM. Orbital exenteration with spontaneous granulation. Arch Ophthalmology 1986;104:139-40.

19 Gorlin RJ, Goltz RW. Multiple nevoid basal-cell epithelioma, jaw cyst and bifid rib. A syndrome. N Engl J Med 1960;262:908-12.

20 Robins $\mathrm{P}$, Rodriguez-Sains R, Rabinovitz H, et al. Moh's surgery for periocular basal carcinomas. J Dermatol Surg Oncol 1985;11:1203.

21 Pach JM, Robertson DM, Taney BS, et al. Prognostic factors in choroidal and ciliary body melanomas with extrascleral extension. Am J Ophthalmol 1986;101:325-31.

22 Rini FJ, Jackobiec FA, Hornblass A, et al. The treatment of advance choroidal melanoma with massive orbital extension. Am J Ophthalmol 1987; 104:634.

23 Shammas HF, Blodi FC. Orbital extension of choroidal melanomas. Arch Ophthalmol 1977;95:2002-5. 
24 Liarikos S, Rapidis AD, Roumeliotis A, et al Secondary orbital melanomas: analysis of 15 cases. J Craniomaxillofac Surg 2000;28:148-52

25 Augsburger JJ, Schneider S, Narayana A, et al. Plaque radiotherapy for choroidal and ciliochoroidal melanomas with limited nodular extrascleral extension. Can J Ophthalmol 2004;39:380-7.

26 Blanco G. Diagnosis and treatment of orbital invasion in uveal melanoma. Can J Ophthalmol 2004;39:388-96.

27 Kersten RC, Tse DT, Anderson RL, et al. The role of orbital exenteration in choroidal melanoma with extrascleral extension. Ophthalmology 1985;92:436-43

28 McLean IW, Jackobiec FA, Zimmerman LE, et al. Tumours of the eye and ocular adnexa. Washington: Maryland Armed Forces Institute of Pathology, $1993 ; 3: 28-35$
29 Zurcher M, Hintschich CR, Garner A, et al. Sebaceous carcinoma of the eyelid: a clinicopathological study. Br J Ophthalmol 1998;82:1049-55.

30 Doxanas MT, Green WR. Sebaceous gland carcinoma: a review of 40 cases. Arch Ophthalmol 1984;102:245-9.

31 Boniuk M, Zimmerman LE. Sebaceous carcinoma of the eyelid, eyebrow, caruncle and orbit. Trans Am Acad Ophthalmol Otolarngol 1968;72:619-41.

32 Ni C, Kuo PK. Mebomian gland carcinoma: a clinicopathologic study of 156 cases with long-period follow-up of 100 cases. Jpn J Ophthalmol 1979:23:388-401

33 Rao NA, Hidayat AA, McLean JW, et al. Sebaceous carcinoma of the ocular adnexa. Hum Pathol 1982;13:113-22.

34 Muqit MMK, Roberts F, Lee WR, et al. Improved survival rates in sebaceous carcinoma of the eyelid. Eye 2004;18:49-53. 